\title{
Errors in estimation of the input signal for integrate-and-fire neuronal models
}

\author{
Enrico Bibbona* \\ INRIM-Istituto Nazionale di Ricerca Metrologica, Strada delle Cacce, 91-10135 Torino, Italy \\ and Department of Mathematics, University of Torino, Via Carlo Alberto 10, 10123 Torino, Italy \\ Petr Lansky ${ }^{\dagger}$ \\ Institute of Physiology, Academy of Sciences of the Czech Republic Videnska 1083, 14220 Prague 4, Czech Republic \\ Laura Sacerdote \\ Department of Mathematics, University of Torino, Via Carlo Alberto 10, 10123 Torino, Italy \\ and NIT-Neuroscience Institute of Torino, Via Accademia Albertina 13, 10123 Torino, Italy \\ Roberta Sirovich ${ }^{\S}$ \\ Department of Mathematics, University of Torino, Via Carlo Alberto 10, 10123 Torino, Italy
}

(Received 19 December 2007; published 24 July 2008)

\begin{abstract}
Estimation of the input parameters of stochastic (leaky) integrate-and-fire neuronal models is studied. It is shown that the presence of a firing threshold brings a systematic error to the estimation procedure. Analytical formulas for the bias are given for two models, the randomized random walk and the perfect integrator. For the third model considered, the leaky integrate-and-fire model, the study is performed by using Monte Carlo simulated trajectories. The bias is compared with other errors appearing during the estimation, and it is documented that the effect of the bias has to be taken into account in experimental studies.
\end{abstract}

DOI: 10.1103/PhysRevE.78.011918

PACS number(s): 87.10.Mn, 02.50.Tt, 87.19.11

\section{INTRODUCTION}

There has been a long-lasting effort dedicated to the mathematical description of single-nerve-cell properties and responses. The models introduced during this process reflect the electrical features of the cell membrane and describe the equivalent electrical circuits. Depending on the physiological details included in the mathematical description, the models range from phenomenological to detailed biophysical models. Models with different degrees of accuracy can be equally important and the choice depends on the task to be undertaken, as well as on the tractability of the equations and the level of resemblance to biological reality [1].

Stochastic (leaky) integrate-and-fire neuronal models are quite simplified representation of physiological properties of cells but still good descriptors of the spiking activity of neurons [2-5]. In addition, the simplification is compensated by the good tractability of the corresponding equations. These models are based on the assumption that the membrane potential is accumulated in time in dependency on the neuronal input. Formally, the membrane potential is described as a stochastic process evolving up to a time when an action potential (spike) is produced, and then the accumulation process starts anew. Thus, the interspike intervals (ISIs) are generated in accordance with a renewal process formed by the first-passage times of the membrane potential to a threshold. The firing is not an intrinsic property of the model; a firing

\footnotetext{
*enrico.bibbona@unito.it

†lansky@biomed.cas.cz

†laura.sacerdote@unito.it

§oberta.sirovich@unito.it
}

threshold and subsequent reset of the process have to be imposed. In other words, the first-passage time of the membrane potential stochastic process through the firing threshold corresponds to the time when the action potential is produced and then the membrane potential is instantaneously reset to its initial value. In this paper we consider three well known models belonging to this category, the randomized random walk (RRW) model, the perfect integrator, also called the Wiener process model, and one of the leaky integrate-and-fire models, also called the OrnsteinUhlenbeck (OU) process model. From the biophysical point of view, only the last-mentioned model can be considered as a compromise between simplification of the biological reality and a good description of the cell activity. However, despite the fact that the two first-mentioned models are oversimplified, their endisputable advantage is their mathematical tractability and thus they serve as examples of the behavior of more sophisticated models.

The integrate-and-fire neuronal models have achieved great popularity, as they show qualitatively many features observed in real neurons [6-9]. However, the validity of any model should also be tested quantitatively by comparison with experimental data, and attempts in this direction can be found in $[2,3]$ where good correspondence with the model is found. The first step for such a comparison is the estimation of the parameters involved. The parameters of the considered models can be divided into two groups [10]. The first group contains parameters characterizing the neuron itself, and such parameters are stable and can be deduced by some indirect methods. These parameters are usually related to the intrinsic properties of the cell and have a direct biological interpretation. Due to such properties they can be determined from measurements that do not involve the application of the model. The parameters from the second group are deter- 
mined by the properties of the input signal to the cell. These parameters may vary rapidly and they can be estimated only by assuming that the membrane potential obeys the equation of the given model. Estimation procedures for these parameters are investigated in this paper, while the parameters characterizing the neuron are considered to be known. The problem can be classified, from a neuronal coding point of view, as signal identification. Other applications in all fields where the first-passage time problem is studied are straightforward.

From an experimental point of view, two types of recording of the electrical activity of the nerve cell can be obtained. The sequence of ISIs, the so-called spike train, can be extracted from extracellular recordings of the field potential. It corresponds, in the considered models, to the sequence of the first-passage times through the firing threshold of the stochastic process describing the membrane potential. Thus, from a statistical point of view, the problem is to estimate the parameters of a stochastic process given the sample of its first-passage times through a threshold. The solution of this problem is well known for the Wiener model and has been applied to neuronal data many times (comparison of the ISI histogram with an inverse Gaussian distribution). On the other hand, for the leaky integrate-and-fire model, it is a mathematically complex task and only a few attempts have been dedicated to this question [11-15]. Using intracellular electrodes, the membrane depolarization can be recorded during ISIs. It corresponds to sampling of trajectories of the stochastic process, and the statistical problem is to estimate the parameters from this type of data. This has been extensively studied in the statistical literature [16], and also with some applications in neuroscience [17-19]. However, in these applications it has never been noticed that the presence of the threshold brings a systematic error to the estimation procedure. As described above, the trajectories of the process are conditioned to take values that are below the firing threshold and finally they are absorbed, meaning that they terminate the first time they hit the threshold. The processes in the presence and absence of the absorbing threshold have different probabilistic characterizations. The problem of estimating the parameters of randomly stopped diffusion processes has been studied in a theoretical framework in $[20,21]$. Here we show that, if the parameters of integrateand-fire neuronal models in the presence of a threshold are estimated by means of estimators derived for models in the absence of a threshold, the estimates are biased. The bias of the estimators is studied for each of the signal parameters involved.

\section{MODELS}

In this section we summarize the basic properties of three well-known models of the neuronal activity, namely, the socalled RRW model and the Wiener process model, both of them belonging to the class denoted as perfect integrators, and the OU process model, which belongs among the socalled leaky integrators (see [6]). These models assume that the membrane potential evolves in time as a realization of a stochastic process until it reaches a fixed threshold $S$ for the first time. In that instant the cell generates an action potential and the membrane potential is reset to a constant, the resetting potential $v_{0}$. The renewal point process given by the first-passage times of the membrane potential to the threshold describes the spiking activity. Formally, let $V=\left\{V_{t}, t\right.$ $\geqslant 0\}$ with $V_{0}=v_{0}$ be the membrane potential stochastic process and let

$$
T=\inf \left\{t \geqslant 0: V_{t} \geqslant S, v_{0} \leqslant S\right\}
$$

be the first-passage time to the threshold $S$. The distribution of the random variable $T$ is the theoretical counterpart of the ISI distribution.

\section{A. Randomized random walk model}

In this model it is assumed that the membrane potential changes according to the mutually independent excitatory and inhibitory inputs received by the cell from the surrounding network of neurons and that both inputs are formed by Poisson processes. Thus, the number of excitatory pulses in the time interval $(0, t]$ is given by a Poisson process with intensity $\lambda^{+}$, denoted by $N_{t}^{+}$, and analogously the number of inhibitory pulses up to time $t$ is denoted by $N_{t}^{-}$, which is a Poisson process with rate $\lambda^{-}$. Thus, from a rate coding point of view, the rates $\lambda^{+}$and $\lambda^{-}$represent the intensities of the input signals. It is assumed that excitatory and inhibitory pulses evoke membrane potential jumps of size $a$, positive, and $i$, negative, respectively. For the sake of simplicity we consider $a=-i$. Thus the membrane potential at time $t$ is given by one of the two following equivalent equations (see [6]):

$$
V_{t}=a\left(N_{t}^{+}-N_{t}^{-}\right) \quad \text { or } \quad V_{t}=\sum_{i=0}^{N_{t}} X_{i},
$$

with $V_{0}=v_{0}, N_{t}$ is a pooled Poisson process with rate $\lambda^{+}$ $+\lambda^{-}$, and $X_{i}$ are independent random variables such that $\mathbb{P}\left(X_{i}=a\right)=\lambda^{+} /\left(\lambda^{+}+\lambda^{-}\right), \mathbb{P}\left(X_{i}=-a\right)=\lambda^{-} /\left(\lambda^{+}+\lambda^{-}\right)$, and $X_{0}=v_{0}$. For the sake of simplicity, the resetting potential $v_{0}$ is put to $v_{0}=0$. Then process (2) is a model for membrane depolarization (the difference between the resting potential and the membrane potential). It is easy to verify that the mean and variance of the membrane potential at time $t$ are

$$
\begin{gathered}
\mathbb{E}\left(V_{t}\right)=a\left(\lambda^{+}-\lambda^{-}\right) t, \\
\operatorname{Var}\left(V_{t}\right)=a^{2}\left(\lambda^{+}+\lambda^{-}\right) t .
\end{gathered}
$$

We refer to the parameter $m=\lambda^{+}-\lambda^{-}$as the net input excitation, since this difference is related to the drift of the membrane potential resulting from the incoming inputs. From (3) we also see that $s^{2}=\lambda^{+}+\lambda^{-}$is related to the variability of the membrane potential and we call it the net input variance. Hence both $m$ and $s^{2}$ characterize the input signal. We denote $\alpha=S / a$, and for simplicity we assume it is an integer number. Notice that $\alpha$ is the minimum number of jumps required to reach the threshold. The distribution of the membrane potential $V_{t}$ in the absence of a firing threshold is known (see [6]). If $\lambda^{+} \geqslant \lambda^{-}$the passage through the threshold $S$ is a sure event and the probability density $g_{S}$ of the first-passage time $T$ is 


$$
g_{S}(t)=\alpha\left(\frac{\lambda^{+}}{\lambda^{-}}\right)^{\alpha / 2} \frac{e^{-\left(\lambda^{+}+\lambda^{-}\right) t}}{t} I_{\alpha}\left(2 t \sqrt{\lambda^{+} \lambda^{-}}\right), \quad t>0,
$$

where $I_{k}(\cdot)$ is the modified Bessel function (see [22]). If $\lambda^{+}$ $>\lambda^{-}$, the expected value and variance of the ISI distribution are the following:

$$
\mathbb{E}(T)=\frac{\alpha}{\left(\lambda^{+}-\lambda^{-}\right)}, \quad \operatorname{Var}(T)=\alpha \frac{\left(\lambda^{+}+\lambda^{-}\right)}{\left(\lambda^{+}-\lambda^{-}\right)^{3}}
$$

for details, see [6].

\section{B. Wiener process model}

Let us introduce the Wiener process model as the diffusion limit of the RRW model. Let $\left\{V_{t}^{n}\right\}_{n \in \mathbb{N}}$ be a sequence of RRW processes such that the amplitudes $a_{n}$ of the jumps decrease to zero as $n \rightarrow+\infty$, while $\lambda_{n}^{+}$and $\lambda_{n}^{-}$increase to infinity in such a way that the means $a_{n}\left(\lambda_{n}^{+}-\lambda_{n}^{-}\right)$and the variances $a_{n}^{2}\left(\lambda_{n}^{+}+\lambda_{n}^{-}\right)$tend to the finite values $\mu$ and $\sigma^{2}$ respectively. Such a sequence of stochastic processes can be shown (see $[6,23]$ ) to converge weakly to a Wiener process $V_{t}$ with parameters $\mu$ and $\sigma^{2}$. The membrane depolarization $V_{t}$ obeys the following equation:

$$
V_{t}=\mu t+\sigma W_{t}, \quad V_{0}=0,
$$

where $W=\left\{W_{t}, t \geqslant 0\right\}$ is the standard Brownian motion and the resetting potential is set to zero with a simple translation of the vertical axis. The parameters $\mu$ and $\sigma^{2}$ are called the drift and infinitesimal variance. As follows from the model construction, both of them describe the effect of the inputs coming into the cell, and they replace $m$ and $s^{2}$ in the previous model.

For $\mu \geqslant 0$ the passage through the threshold $S$ is a sure event and the ISI distribution, that is, the density of the random variable $T$, is given by the equation

$$
g_{S}(t)=\frac{S}{\sqrt{2 \pi \sigma^{2} t^{3}}} \exp \left(-\frac{(S-\mu t)^{2}}{2 \sigma^{2} t}\right) .
$$

It can be obtained as the diffusion limit of Eq. (4) (see the Appendix). According to the behavior expected from (5) and the diffusion limit conditions on $m$ and $s^{2}$, the mean value of $T$ (mean ISI) and its variance, for $\mu>0$, are given by

$$
\mathbb{E}(T)=\frac{S}{\mu}, \quad \operatorname{Var}(T)=\frac{S \sigma^{2}}{\mu^{3}} .
$$

\section{Ornstein-Uhlenbeck model}

This model belongs to the class of leaky integrate-and-fire models. It is a generalization of the Wiener process model that takes into account the spontaneous decay of the membrane potential to the resting level in the absence of inputs. It describes the membrane potential as an OU process, given by the following stochastic differential equation:

$$
d V_{t}=\left[-\beta\left(V_{t}-v_{0}\right)+\mu\right] d t+\sigma d W_{t}, \quad V_{0}=v_{0},
$$

where $W$ is again the standard Brownian motion. Equation (9) is usually transformed into an equation for the membrane depolarization $V_{t}-v_{0}$ and thus takes the form

$$
d V_{t}=\left(-\beta V_{t}+\mu\right) d t+\sigma d W_{t}, \quad V_{0}=0,
$$

where both, the resetting potential and the resting potential are equal to zero. The parameter $1 / \beta$ is the membrane time constant and we suppose it is known. The parameters $\mu$ and $\sigma^{2}$ in Eq. (10) have the same meaning as in Eq. (6).

Equation (10) defines a Gaussian process with

$$
\mathbb{E}\left(V_{t}\right)=\frac{\mu}{\beta}-\frac{\mu}{\beta} e^{-\beta t},
$$

$$
\operatorname{Var}\left(V_{t}\right)=\frac{\sigma^{2}}{2 \beta}\left(1-e^{-2 \beta t}\right) .
$$

Hence the transition density function of the process (10) in the absence of the threshold is the following:

$$
\begin{aligned}
& f(x, t \mid y, s) \frac{1}{\sqrt{\left(\pi \sigma^{2} / \beta\right)\left(1-e^{-2 \beta(t-s)}\right)}} \\
& \quad \times \exp \left(-\frac{\left(x-\mu / \beta+(\mu / \beta-y) e^{-\beta(t-s)}\right)^{2}}{\left(\sigma^{2} / \beta\right)\left(1-e^{-2 \beta(t-s)}\right)}\right) .
\end{aligned}
$$

The first-passage time of the process (10) to a constant threshold cannot be calculated in a closed form and just its Laplace transform is known (see [24]). However, the moments can be analytically calculated (see [25]), and the mean first-passage time is given by (see [26])

$$
\mathbb{E}(T)=\frac{\sqrt{\pi}}{\beta} \int_{-\mu / \sqrt{\beta} \sigma}^{(S \beta-\mu) / \sqrt{\beta} \sigma}[1+\operatorname{erf}(x)] e^{x^{2}} d x,
$$

where $\operatorname{erf}(\cdot)$ is the error function. The second-order moment is explicitly given in $[27,28]$.

\section{SIGNAL ESTIMATION IN THE ABSENCE OF THE FIRING THRESHOLD}

In this section we recall results on the estimate of the input parameters from samples with no threshold condition imposed.

\section{A. Sampling strategy}

We assume that the process $V_{t}$ is observed at regularly spaced discrete instants. The sample is the sequence $\left(V_{i}\right)_{i=1}^{n}$, that is, one trajectory of the process $V_{t}$ sampled at $n$ time points $t_{i}=i h$, for $i=1, \ldots, n$. The value of $n$ is selected independently of the behavior of the process $V_{t}$, in contrast to the approach in the next section. Let us remark that, for the RRW process, we need to suppose that the constant step of sampling $h$ is sufficiently small to guarantee that the probability to have more than one event in the time interval $[t, t$ $+h$ ) is negligible for all $t$. This condition on $h$ enables us to assume that from the sample $\left(V_{i}\right)_{i=1}^{n}$ we can deduce exactly the number of events in the processes $N_{t}^{+}$and $N_{t}^{-}$.

The estimators considered throughout the paper apply to the sample described above, i.e., made of just one discretely observed trajectory of the process. However, if multiple trajectories are available, the estimates from each trajectory can be averaged so that the standard deviation of the estimate is 
reduced by a factor $1 / \sqrt{N}$, where $N$ is the number of trajectories. For a detailed example of the application of such estimators to experimental data, see [18].

\section{B. Randomized random walk model}

The unknown parameters are the intensities $\lambda^{+}$and $\lambda^{-}$of the processes $N_{t}^{+}$and $N_{t}^{-}$. From Eq. (3), we see that these parameters are involved in the mean and variance of the process as $m=\lambda^{+}-\lambda^{-}$and $s^{2}=\lambda^{+}+\lambda^{-}$. Moreover, their sum and difference have a clear interpretation in the construction of the Wiener process as the diffusion limit of the RRW process. To maintain such a correspondence, we consider here estimators for $m$ and $s^{2}$ and we denote them by $\hat{m}$ and $\hat{s}^{2}$, respectively.

Recalling that $\lambda^{+}$and $\lambda^{-}$are the intensity parameters of the Poisson processes $N_{t}^{+}$and $N_{t}^{-}$, and that $N_{t}^{+}$and $N_{t}^{-}$are independent, we obtain the maximum likelihood estimator

$$
\hat{m}=\frac{N_{t_{n}}^{+}-N_{t_{n}}^{-}}{t_{n}}=\frac{V_{n}}{a t_{n}},
$$

where the second equality follows from definition of the process $V_{t}$, Eq. (2). The same arguments lead to the following equation:

$$
\hat{s}^{2}=\frac{N_{t_{n}}^{+}+N_{t_{n}}}{t_{n}}=\frac{\sum_{i=1}^{n}\left(V_{i}-V_{i-1}\right)^{2}}{a^{2} t_{n}},
$$

where the second equality holds if $h$ is sufficiently small to have at most one jump in any time interval of length $h$. When $V_{i}-V_{i-1}$ is different from zero, it means that we had an event in $N_{t}^{+}$or $N_{t}^{-}$, and the sum of all the differences gives the number of events in $N_{t}$ up to the last time of observation $t_{n}$. It can be easily shown that both estimators (14) and (15) are unbiased.

\section{Wiener process model}

Estimators for the unknown parameters $\mu$ and $\sigma^{2}$ can be derived by the maximum likelihood method, as for example in $[16,17]$. The estimator for $\mu$ is given by

$$
\hat{\mu}=\frac{\sum_{i=1}^{n}\left(V_{i}-V_{i-1}\right)}{t_{n}}=\frac{V_{n}}{t_{n}} .
$$

Let us remark that estimator (16), due to the convergence of the RRW process to the Wiener process, could be obtained as the diffusion limit of estimator $a \hat{m}$ [compare with Eq. (14)]. The estimator for $\sigma^{2}$, supposing $\mu$ known, is given by the expression

$$
\hat{\sigma}^{2}=\frac{1}{t_{n}} \sum_{i=1}^{n}\left(V_{i}-V_{i-1}-\mu h\right)^{2} .
$$

If $\mu$ is unknown, we can substitute $\mu$ with $\hat{\mu}$ in the preceding equation. It can be proved that the estimators $\hat{\mu}$ and $\hat{\sigma}^{2}$ are unbiased.
If $h$ is sufficiently small we can estimate the variance $\sigma^{2}$ by means of the following estimator, obtained by ignoring the term $\mu h$ in Eq. (17):

$$
\hat{\sigma}_{\text {approx }}^{2}=\frac{1}{t_{n}} \sum_{i=1}^{n}\left(V_{i}-V_{i-1}\right)^{2} .
$$

Indeed, estimator (18) has the bias $\mu^{2} h / n$, which for small $h$ and large $n$ can be disregarded. The advantage in introducing such an approximation relies on the fact that $\hat{\sigma}_{\text {approx }}^{2}$ in (18) is the diffusion limit of the corresponding estimator (15) for the RRW model multiplied by $a^{2}$. This property will be used later on.

\section{Ornstein-Uhlenbeck process model}

The maximum likelihood estimator for the unknown parameters of the OU process can be found in [16] and in the neuronal context were introduced in [17]. The log-likelihood function for the OU process with transition probability density function (12) is given by

$$
\begin{aligned}
\mathrm{L}\left(V_{1}, \ldots, V_{n} \mid \mu, \sigma^{2}\right)= & -\frac{n}{2} \log \left(\frac{\pi \sigma^{2}}{\beta}\left(1-e^{-2 \beta h}\right)\right) \\
& -\sum_{i=1}^{n} \frac{\left[V_{i}-\mu / \beta+\left(\mu / \beta-V_{i-1}\right) e^{-\beta h}\right]^{2}}{\left(\sigma^{2} / \beta\right)\left(1-e^{-2 \beta h}\right)} .
\end{aligned}
$$

Assuming $\beta$ known, we can solve the likelihood equation exactly, and the resulting estimator for the drift parameter $\mu$ is the following:

$$
\hat{\mu}=\frac{\sum_{i=1}^{n} V_{i}-e^{-\beta h} \sum_{i=1}^{n} V_{i-1}}{(n / \beta)\left(1-e^{-\beta h}\right)}=\frac{V_{n}}{(n / \beta)\left(1-e^{-\beta h}\right)}+\frac{\beta}{n} \sum_{i=1}^{n-1} V_{i} .
$$

The estimator for the diffusion coefficient, if $\mu$ is known, is given by

$$
\hat{\sigma}^{2}=\frac{2}{n} \sum_{i=1}^{n} \frac{\left[V_{i}-\mu / \beta+\left(\mu / \beta-V_{i-1}\right) e^{-\beta h}\right]^{2}}{(1 / \beta)\left(1-e^{-2 \beta h}\right)} .
$$

To get the estimator for the diffusion coefficient when $\mu$ is not known, we can substitute (20) in the preceding equation and add a factor to correct an asymptotic bias:

$$
\hat{\hat{\sigma}}^{2}=\frac{2}{n-1} \sum_{i=1}^{n} \frac{\left[V_{i}-\hat{\mu} / \beta+\left(\hat{\mu} / \beta-V_{i-1}\right) e^{-\beta h}\right]^{2}}{(1 / \beta)\left(1-e^{-2 \beta h}\right)} .
$$

It can be proved that the estimators (20)-(22) are unbiased.

\section{SIGNAL ESTIMATION IN THE PRESENCE OF THE FIRING THRESHOLD}

In this section we show that if we estimate the parameters in the presence of a firing threshold by means of estimators that are formally equivalent to those introduced in the previ- 
ous section, we get biased estimates for some of the parameters involved. Let us remark that the processes in the presence of the firing threshold terminate at $S$ the first time they hit it. Hence at any time their trajectories are conditioned to take values that are below the threshold. We could say that the process $V_{t}$ and the conditioned process, which we denote as $V_{t}^{a}$, are truly different processes. They have different distributions at any time, different transition probability density functions, and hence different likelihood functions (in the case of the Wiener process all the analytical expressions are known for both processes; see, for example, [24]). As a consequence, unbiased maximum likelihood estimators for the process $V_{t}$ do not necessarily maintain their properties when applied to a sample drawn from the conditioned process $V_{t}^{a}$.

\section{A. Sampling strategy}

We assume the same sampling procedure as in the previous section. Hence the sample is one discretely observed trajectory of the process $\left(V_{i}^{a}\right)_{i=1}^{n}$. The last time point of the sampling $t_{n}=n h$ is a realization of the first-passage time $T$ of the considered process, and thus the value of the process at that time is $V_{n}^{a}=S$ (hence the sample is drawn from one ISI). In this case the size of the sample cannot be arbitrarily fixed, but is given by the random variable $K=[T / h]$, where by [ ] we denote the integer part. As in Sec. III, we suppose $h$ sufficiently small to guarantee that the probability to have more than one event in the time interval $[t, t+h)$ is negligible for all $t$. Again, if more than one ISI is available, the estimates from each sampled trajectory can be averaged, reducing the variability.

\section{B. Randomized random walk}

As assumed, $S / a=\alpha$ is an integer number. Given that $t_{K}$ $=T$ and that $V_{K}^{a}=S$, estimators (14) and (15) become

$$
\begin{gathered}
\hat{m}_{a}=\frac{\alpha}{T}, \\
\hat{s}_{a}^{2}=\frac{N_{T}^{+}+N_{T}^{-}}{T}=\frac{\sum_{i=1}^{K}\left(V_{i}^{a}-V_{i-1}^{a}\right)^{2}}{a^{2} T} .
\end{gathered}
$$

Using Eq. (4), the expected value of the estimator (23) is given by

$$
\mathbb{E}\left(\hat{m}_{a}\right)=\alpha^{2}\left(\frac{\lambda^{+}}{\lambda^{-}}\right)^{\alpha / 2} 2 \sqrt{\lambda^{+} \lambda^{-}} \int_{0}^{+\infty} z^{-2} e^{-\left[\left(\lambda^{+}+\lambda^{-}\right) / 2 \sqrt{\lambda^{+} \lambda^{-}}\right] z} I_{\alpha}(z) d z
$$

where $I .(\cdot)$ is the modified Bessel function (see [22]). Analogous computations lead to the following expression for the second moment of (23):
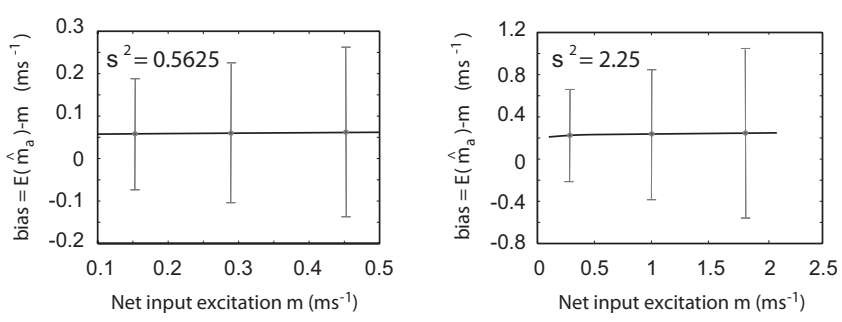

FIG. 1. Bias of estimator (23) versus the net input excitation $m$, with error bars $\left[\mp \mathcal{S}\left(\hat{m}_{a}\right)\right.$, where $\mathcal{S}$ is the standard deviation; cf. (26)], for two different values of $s^{2}=0.5625$ (left panel) and $2.25 \mathrm{~ms}^{-1}$ (right panel). Other parameters are $a=1 \mathrm{mV}$ and $S$ $=10 \mathrm{mV}$.

$$
\mathbb{E}\left(\hat{m}_{a}^{2}\right)=\alpha^{3}\left(\frac{\lambda^{+}}{\lambda^{-}}\right)^{\alpha / 2} 4\left(\lambda^{+} \lambda^{-}\right) \int_{0}^{+\infty} z^{-3} e^{-\left[\left(\lambda^{+}+\lambda^{-}\right) / 2 \sqrt{\lambda^{+} \lambda^{-}}\right] z} I_{\alpha}(z) d z
$$

The integrals that appear in (25) and (26) cannot be calculated analytically, but from their numerical evaluation it is clear that estimator (23) is biased.

The results are illustrated by means of Figs. 1 and 2. In Fig. 1 we plot the bias of estimator $(23)$, i.e. $\mathbb{E}\left(\hat{m}_{a}\right)-m$, versus the net input excitation $m$. The bias is positive, constant with respect to $m$, and increasing with $s^{2}$ (note that the panels have different scales). The standard deviation of the estimates increases in both $m$ and $s^{2}$. The bias increases as $a$ increases (not illustrated).

In Fig. 2 we illustrate the effect of the overestimation of $m$ on the transfer function. Let us recall that the transfer function is the curve that plots the output firing frequency $f$ $=1 / \mathbb{E}(T)$ versus the net input excitation, see, for example, [29]. In Fig. 2 two kinds of transfer functions are given. The theoretical transfer function (dash-dotted line) plots the points $(m, f(m))$, where $f(m)$ is computed according to Eq.

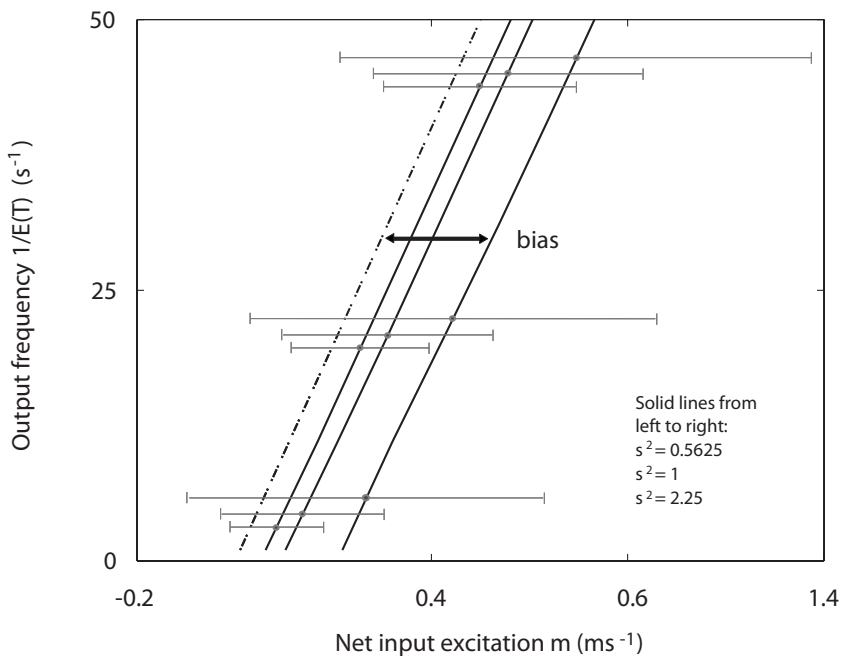

FIG. 2. Transfer functions of the RRW model, $a=1, S=10 \mathrm{mV}$. Dash-dotted line: theoretical transfer function. Solid lines: experimental transfer function for the mean estimated values of the net input excitation with error bars $\left[\mp \mathcal{S}\left(\hat{m}_{a}\right)\right]$ for three different values of $s^{2}=0.5625,1$, and $2.25 \mathrm{~ms}^{-1}$. 
(5) as $f(m)=m / \alpha$. The experimental transfer function (continuous lines, for three values of $s^{2}$ ) plots the points $\left(\mathbb{E}\left(\hat{m}_{a}\right), f(m)\right)$. We call it experimental since from the experimental viewpoint the net input excitation is estimated from the sample. Since it is overestimated, as shown in Fig. 1, the experimental transfer function is shifted to the right and the amount of the shift is exactly the bias.

To calculate the expected value of estimator (24) let us recall that the probability $P_{k}$ that the first-passage time through the threshold $S$ comes at the $k$ th jump, for $k \geqslant \alpha$, and $\alpha$ and $k$ with the same parity, is given by (cf. [30], Chap. 2, formula 7.11)

$$
P_{k}=\frac{\alpha}{k}\left(\begin{array}{c}
k \\
\frac{\alpha+k}{2}
\end{array}\right)\left(\frac{\lambda^{+}}{\lambda^{+}+\lambda^{-}}\right)^{(\alpha+k) / 2}\left(\frac{\lambda^{-}}{\lambda^{+}+\lambda^{-}}\right)^{(\alpha-k) / 2}
$$

Moreover, the time of the $k$ th event in a Poisson process of parameter $s^{2}=\lambda^{+}+\lambda^{-}$is $\Gamma\left(k, s^{2}\right)$ distributed with density $f^{\Gamma}(t)$. It follows that the probability that the first passage occurs at the $k$ th jump and at time $t$ can be calculated by means of the density $P_{k} f^{\Gamma}(t)$.

Since $k \geqslant \alpha$ and has the same parity, let us write it as $k$ $=\alpha+2 \nu, \nu \geqslant 0$. The expected value of (24) for $\alpha \neq 1$ is thence

$$
\begin{aligned}
\mathbb{E}\left(\hat{s}_{a}^{2}\right)= & \int_{0}^{+\infty} \sum_{\nu=0}^{+\infty} \frac{\alpha+2 \nu}{t} P_{\alpha+2 v} f^{\Gamma}(t) d t \\
= & \sum_{\nu=0}^{+\infty}(\alpha+2 \nu) P_{\alpha+2 \nu} \int_{0}^{+\infty} \frac{f^{\Gamma}(t)}{t} d t \\
= & \sum_{\nu=0}^{+\infty} \alpha\left(\begin{array}{c}
\alpha+2 \nu \\
\alpha+\nu
\end{array}\right)\left(\frac{\lambda^{+}}{\lambda^{+}+\lambda^{-}}\right)^{\alpha+\nu}\left(\frac{\lambda^{-}}{\lambda^{+}+\lambda^{-}}\right)^{\nu} \frac{\lambda^{+}+\lambda^{-}}{\alpha+2 \nu-1} \\
= & \frac{\alpha}{\alpha-1}\left(\frac{\lambda^{+}}{\lambda^{+}+\lambda^{-}}\right){ }^{\alpha}\left(\lambda^{+}+\lambda^{-}\right) F\left(1+\frac{\alpha}{2},-\frac{1}{2}+\frac{\alpha}{2} ; 1\right. \\
& \left.+\alpha ; \frac{4 \lambda^{+}}{\lambda^{+}+\lambda^{-}}\left(1-\frac{\lambda^{+}}{\lambda^{+}+\lambda^{-}}\right)\right) \\
= & \frac{\alpha}{\alpha^{2}-1}\left[\left(\lambda^{+}-\lambda^{-}\right)+\alpha\left(\lambda^{+}+\lambda^{-}\right)\right]=\frac{\alpha}{\alpha^{2}-1}\left(m+\alpha s^{2}\right)
\end{aligned}
$$

where $F(\cdot, \cdot ; \cdot ; \cdot)$ is the hypergeometric function, and the equality between the last two lines is due to formulas 15.1.13, 15.2.21, and 15.2.26 in [22]. If $\alpha=1$ the estimator (24) has infinite expectation. Indeed, for $\alpha=1$ and $\nu=0$, the function $f^{\Gamma}$ reduces to the density of an exponential random variable and the integral in Eq. (28) diverges. Note that this term accounts for those trajectories that are absorbed at the first jump for which the first-passage time is too short to let the integral converge. The bias is linear in both $m$ and $s^{2}$ and vanishes for $\alpha \rightarrow \infty$.

Analogous computations allow us to calculate the second moment of (24) and the variance, for $\alpha \neq 1,2$, is

$$
\begin{aligned}
& \operatorname{Var}\left(\hat{s}_{a}^{2}\right) \\
& =\frac{\left(3 \alpha^{4}-4 \alpha^{2}+4\right) m^{2}+\left(\alpha^{5}+5 \alpha^{3}\right) m s^{2}+\left(\alpha^{4}+6 \alpha^{2}-4\right) s^{4}}{\left(\alpha^{2}-4\right)\left(\alpha^{2}-1\right)^{2}} .
\end{aligned}
$$

For $\alpha=1,2$ the variance diverges.

In order to get some insight into the results illustrated above, let us examine two special cases where the presence of biases could be guessed without calculations. Consider a RRW with $m=0$. Since the passage through the threshold is a sure event, the estimator (23) for $m$ is well defined and strictly positive. Hence it has a positive bias. In order to understand why the estimate of $s^{2}$ is biased too, consider the case with only upward jumps $\left(\lambda^{-}=0\right)$. In this case $m=s^{2}$ and $N_{T}^{+}=\alpha$. Therefore estimators (23) and (24) are identical: since $\hat{m}_{a}$ is positively biased, so is $\hat{s}_{a}^{2}$. Let us finally observe that if $\alpha$ is large the threshold is far away with respect to the amplitude of the jumps and, since few trajectories undergo a fast absorption, the biases are very small.

\section{Wiener process model}

For the Wiener model in the presence of a threshold the estimator (16) for the parameter $\mu$ becomes

$$
\hat{\mu}_{a}=\frac{S}{T}
$$

and the approximated estimator (18) for $\sigma^{2}$ under the condition $\mu^{2} h \ll \sigma^{2}$ becomes

$$
\hat{\sigma}_{\text {approx }, a}^{2}=\frac{1}{T} \sum_{i=1}^{K}\left(V_{i}^{a}-V_{i-1}^{a}\right)^{2},
$$

where $K=[T / h]$. As already shown in [21], the estimator (31) is biased. In fact,

$$
\mathbb{E}\left(\hat{\mu}_{a}\right)=\mu+\frac{\sigma^{2}}{S} .
$$

The bias does not depend on $\mu$, whereas it is linear in $\sigma^{2}$; thus it becomes relatively more relevant for small $\mu$ and large $\sigma^{2}$. We can observe that, as long as the first-passage time density of the RRW model converges in the diffusion limit to that of the Wiener process model, the two estimators (23) and (31) converge and so do the expectations [compare formulas (25) and (33)]. The variance of $\hat{\mu}_{a}$ can be calculated by direct integration of the first-passage time density (7) and we have

$$
\operatorname{Var}\left(\hat{\mu}_{a}\right)=\frac{\sigma^{2}}{S^{2}}\left(S \mu+2 \sigma^{2}\right) .
$$

The transfer functions are completely similar to those of the RRW model.

Although the density of the absorbed Wiener process can be explicitly calculated (see [24]), we cannot compute the expectation of (32) by direct integration. The estimator (32) is the same function of $V^{a}$ as is the estimator (24) of the variance of the RRW model, but multiplied by $a^{2}$. As the two 
processes (2) and (6) converge in the diffusion limit, we can conclude that the expectation of (32) is equal to the limit of the expectation (29). Hence, if we substitute the parameters $m=\lambda^{+}-\lambda^{-}$and $s^{2}=\lambda^{+}+\lambda^{-}$with their asymptotics $m \sim \mu / a$ and $s^{2} \sim \sigma^{2} / a^{2}$ in (29) and multiply by $a^{2}$, we get

$$
\mathbb{E}\left(\hat{\sigma}_{\text {approx }, a}^{2}\right)=\lim _{a \rightarrow 0} a^{2} \mathbb{E}\left(\hat{s}_{a}^{2}\right)=\lim _{a \rightarrow 0} \frac{a^{2} S}{S^{2}-a^{2}} \mu+\frac{S^{2}}{S^{2}-a^{2}} \sigma^{2}=\sigma^{2} .
$$

We conclude that, even if the estimator $\hat{s}_{a}^{2}$ for the absorbed RRW model is biased, by taking its diffusion limit $\hat{\sigma}_{\text {approx }, a}^{2}$, the bias vanishes.

Let us consider the diffusion limits of the two special cases $\left(m=0\right.$ and $\left.\lambda^{-}=0\right)$ presented in the previous section. The example with $m=0$ can be proposed again for the Wiener model with $\mu=0$ and with the same arguments the bias can be guessed. Indeed the first passage is a sure event and, even if $\mathbb{E}(T)$ is not finite, $\mathbb{E}(1 / T) \neq 0$. On the contrary, the case with $\lambda^{-}=0$, which suggested the presence of the bias of $\hat{s}_{a}^{2}$ in the RRW model, in the diffusive limit falls into the degenerate case $\sigma^{2}=0$ and fails to give any insight. Indeed both conditions $\mu \sim a m$ and $\sigma^{2} \sim a^{2} s^{2}$ are required, but $m$ $=s^{2}$.

\section{Ornstein-Uhlenbeck process model}

The estimator (20) for the parameter $\mu$ in the presence of the threshold becomes

$$
\hat{\mu}_{a}=\frac{S}{K / \beta\left(1-e^{-\beta h}\right)}+\frac{\beta}{K} \sum_{i=1}^{K-1} V_{i}^{a},
$$

and the estimators (21) and (22) for $\sigma^{2}$ with $\mu$ known and unknown, respectively, become

$$
\hat{\sigma}_{a}^{2}=\frac{2}{K} \sum_{i=1}^{K} \frac{\left[V_{i}^{a}-\mu / \beta+\left(\mu / \beta-V_{i-1}^{a}\right) e^{-\beta h}\right]^{2}}{1 / \beta\left(1-e^{-2 \beta h}\right)}
$$

and

$$
\hat{\sigma}_{a}^{2}=\frac{2}{K-1} \sum_{i=1}^{K} \frac{\left[V_{i}^{a}-\hat{\mu}_{a} / \beta+\left(\hat{\mu}_{a} / \beta-V_{i-1}^{a}\right) e^{-\beta h}\right]^{2}}{1 / \beta\left(1-e^{-2 \beta h}\right)} .
$$

The first-passage time density and the probability distribution of $V_{t}^{a}$ are not known analytically and the expectation of the estimators (36)-(38) cannot be calculated directly; thus we evaluate them by means of the Monte Carlo method.

For each choice of the parameters, we generate $N$ $=10000$ trajectories, both absorbed and not absorbed. With the $N$ estimates obtained using estimators (20), (21), and (38), we build confidence intervals at the $95 \%$ level for the mean of $\hat{\mu}_{a}$ and the mean of $\hat{\sigma}_{a}^{2}$. The discretization step is fixed to $h=0.01 \mathrm{~ms}$, so that the sample sizes $n$ are sufficiently large in each of the $N$ repetitions and for every considered value of the parameters. The lengths of each pair of absorbed and not absorbed trajectories, and hence the sample sizes, are identical. To ensure this, we always first generate a trajectory until it is absorbed and then generate a trajectory in the absence of the threshold with the same length. In such
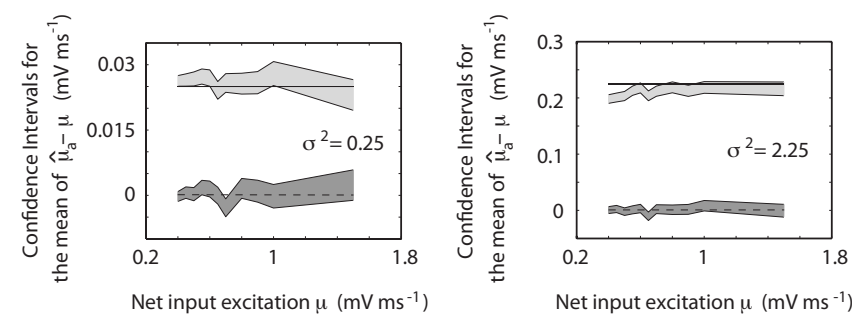

FIG. 3. Confidence intervals at $95 \%$ level for the bias of estimator $\hat{\mu}_{a}$ (36) in the OU process model in dependency on $\mu$ and for two different values of $\sigma^{2}=0.25$ (left panel) and $2.25 \mathrm{mV}^{2} \mathrm{~ms}^{-1}$ (right panel). Dark gray: $\mu$ estimated in the absence of the threshold. Light gray: $\mu$ estimated in the presence of the threshold. Continuous line: $\sigma^{2} / S$, i.e., bias predicted by Eq. (33) holding for a Wiener process with the same parameters. Other parameters are $S$ $=10 \mathrm{mV}, v_{0}=0 \mathrm{mV}, \beta=0.05 \mathrm{~ms}^{-1}$.

a way any property of the estimators that depends upon $n$ affects both estimates equally. The samples are generated using the classical algorithms for discrete time approximations of the solution of stochastic differential equations (see [31]). For the simulation of absorbed trajectories we use the bridge process correction proposed in [32].

The values of the parameters have been chosen in a biologically compatible range. The threshold is fixed at $S$ $=10 \mathrm{mV}$. The net input excitation parameter varies in the range $\mu \in[0.4,1.5] \mathrm{mV} \mathrm{ms}^{-1}$. The diffusion parameter $\sigma^{2}$ varies in the interval $[0.25,2.25] \mathrm{mV}^{2} \mathrm{~ms}^{-1}$. The membrane time constant is fixed at $1 / \beta=20 \mathrm{~ms}$. The chosen range of the parameters covers both the subthreshold $(\mu / \beta<S)$ and suprathreshold regimens $(\mu / \beta>S)$. In particular, the chosen ranges are consistent with the values estimated from biological data in [18].

In Fig. 3 we plot the confidence intervals for the bias of the estimated $\mu$ in the absence (dark gray) and in the presence (light gray) of the threshold. In the presence of the threshold, there is a positive bias, constant in $\mu$ and increasing with $\sigma^{2}$. Let us remark that the bias is very well fitted by the value $\sigma^{2} / S$ given by Eq. (33), which holds for a Wiener process with the same parameters (continuous line in the figure).

The theoretical and experimental transfer functions of the OU process model (10) are plotted in Fig. 4 for two different values of $\sigma^{2}$. Using Eq. (13), as in $[2,33,34]$, the theoretical transfer function plots $1 / \mathbb{E}(T)$ versus $\mu$ and the experimental transfer function plots the firing frequency versus the esti-
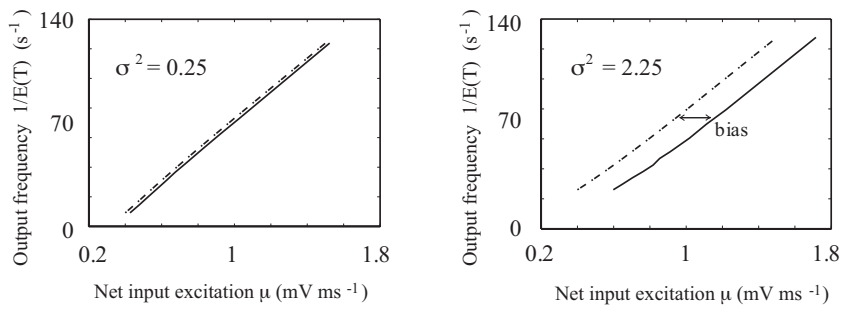

FIG. 4. Transfer functions of the OU process model with $S$ $=10 \mathrm{mV}, \beta=0.05 \mathrm{~ms}^{-1}, v_{0}=0 \mathrm{mV}$, and for two values of $\sigma^{2}$ $=0.25$ and $2.25 \mathrm{mV}^{2} \mathrm{~ms}^{-1}$. Dash-dotted line: theoretical transfer function. Solid line: experimental transfer function. 

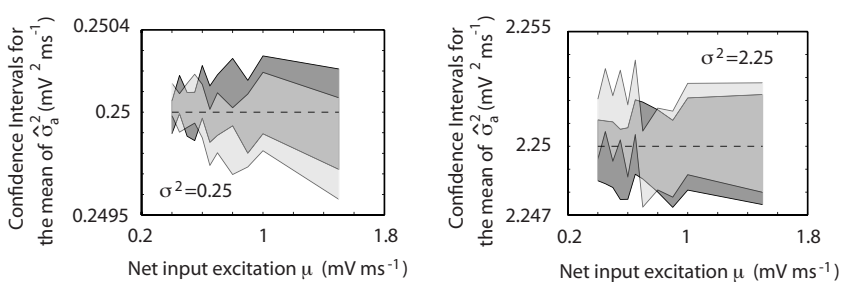

FIG. 5. Confidence interval at 95\% level for the mean of estimator $\hat{\sigma}_{a}^{2}(37)$ in the OU process model in dependency on $\mu$ (known) and for two values of $\sigma^{2}=0.25$ and $2.25 \mathrm{mV}^{2} \mathrm{~ms}^{-1}$. Dark gray: estimated $\sigma^{2}$ in the absence of the threshold (21). Light gray: estimated $\sigma^{2}$ in the presence of absorbing threshold (37). Other parameters are $S=10 \mathrm{mV}, v_{0}=0 \mathrm{mV}, \beta=0.05 \mathrm{~ms}^{-1}$.

mated value of $\mu$ obtained from Eq. (36). Since the estimator (36) is biased, the experimental transfer function is displaced to the right and the bias is exactly the amount of the shift.

In Fig. 5 we plot the confidence intervals for the mean of $\hat{\sigma}_{a}^{2}$ in (37), in dependency on known $\mu$ for different values of $\sigma^{2}$. We compare estimates in the absence of the threshold (21) (dark gray) with estimates from absorbed trajectories (37) (light gray). The estimator (37) seems to be unbiased even for the absorbed trajectories. Very similar graphs are obtained for the estimator of $\sigma^{2}$ with $\mu$ unknown. This result is consistent with that obtained for the Wiener process.

\section{QUANTIFICATION OF THE BIASES AND COMPARISON WITH OTHER SOURCES OF ERROR}

In the previous section we showed that the estimates of some parameters are biased due to the presence of the threshold. In this section we evaluate the magnitude of the biases in dependency on the model and its parameters, and we compare it with other sources of errors. As a measure of statistical variability we consider the standard deviation of the estimate. We also study the errors due to a possible misspecification of one of the parameters that are considered to be known (the ratio $\alpha=S / a$ for the RRW, the threshold $S$ for the Wiener model, and $S$ and $\beta$ for the OU process). To avoid mutual dependencies between these presumably known parameters, we always consider only one wrongly specified parameter at a time. Let us remark that the errors in the estimates we are comparing in this section have quite different character. For example, the statistical variability is not systematic, in contrast with the other considered errors, and it can be reduced by averaging the estimates over many ISIs.

\section{A. Randomized random walk model}

We focus on the parameter $m=\lambda^{+}-\lambda^{-}$. In Fig. 6 we compare the following three quantities: (1) the relative bias (\%) [cf. Eq. (25)]:

$$
100 \times \frac{\mathbb{E}\left(\hat{m}_{a}\right)-m}{m} ;
$$

(2) the relative statistical variability of the estimate [cf. Eq. (26)]:
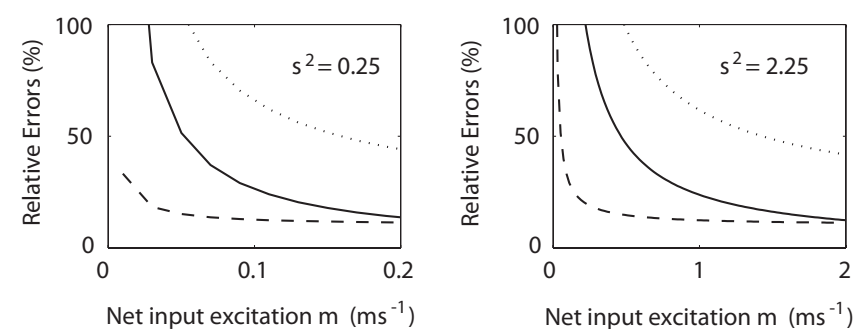

FIG. 6. Relative errors for estimated $m$ in RRW model for two values of $s^{2}=0.25$ and $2.25 \mathrm{~ms}^{-1}$. Solid line: absorption bias. Dotted line: standard deviation. Dashed line: errors introduced by misspecification of $\alpha$ by $10 \% . S=10 \mathrm{mV}, a=1 \mathrm{mV}$.

$$
100 \times \frac{\mathcal{S}\left(\hat{m}_{a}\right)}{m}
$$

and (3) the relative error that is introduced if the value $\alpha$ $+\varepsilon$ is erroneously assigned to the ratio $\alpha=S / a$ calculated according to the formula

$$
\frac{100}{m}\left[\mathbb{E}\left(\hat{m}_{a}(\alpha+\varepsilon)\right)-\mathbb{E}\left(\hat{m}_{a}(\alpha)\right)\right]=\frac{\varepsilon}{\alpha} 100 \times \frac{\mathbb{E}\left(\hat{m}_{a}\right)}{m} .
$$

The errors are presented as functions of $m$ for two different values of $s^{2}$. As illustrated in Fig. 1, the bias is constant in $m$; hence the relative bias has the functional form const $/ \mathrm{m}$. The error introduced by a misspecification of $\alpha$ is proportional to the relative estimate of $m$. If $\hat{m}_{a}$ were unbiased, the error would be constant (equal to $100 \varepsilon / \alpha$ ), but due to the increase of the relative bias for smaller $m$, the error steers toward larger values.

In the considered range of the parameters, the bias due to the absorbing boundary is never negligible. In particular, for small values of $m$ (we considered here values ranging down to $m=0.01 \mathrm{~ms}^{-1}$ ), the relative bias has the same order of magnitude as the relative statistical variability, and they are so important that we cannot get reliable estimates without correcting the bias and averaging over many ISIs. For such values of $m$ the error due to an erroneously assigned $\alpha$ is negligible with respect to the others. As $m$ increases (with the constraint $m \leqslant s^{2}$ ), the relative bias and statistical variability are reduced and settle on a constant value with the same order of magnitude as the error introduced by misspecification of $\alpha$, which for the chosen values of the parameters is $10 \%$.

If $\alpha$ is misspecified by a negative $\varepsilon$, the error keeps the same magnitude but in the opposite direction. The same holds true for misspecification of $S$ in the Wiener and OU models.

\section{B. Wiener model}

We have shown that the estimate of $\sigma^{2}$ is unbiased. On the contrary, the estimate of $\mu$ is affected by absorption bias. To quantify it, we plot in Fig. 7 the analogous quantities as for the RRW model ( $m$ is replaced by $\mu$ ). The explicit expressions for all the considered relative errors are easily derived 

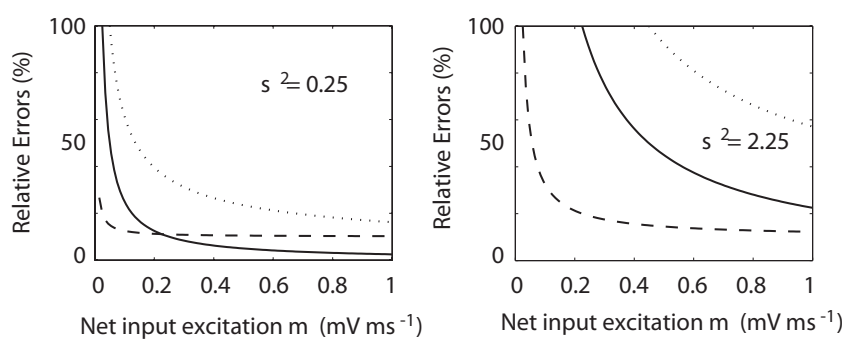

FIG. 7. Relative errors for estimated $\mu$ in the Wiener process model for two values of $\sigma^{2}=0.25$ and $2.25 \mathrm{mV}^{2} \mathrm{~ms}^{-1}$. Solid line: absorption bias. Dotted line: standard deviation. Dashed line: misspecification of $S$ by $10 \%$. $S=10 \mathrm{mV}$.

from Eqs. (33) and (34). In particular, since the bias is constant in $\mu$ and equal to $\sigma^{2} / S$, the relative absorption bias is $100 \sigma^{2} /(S \mu)$.

Again, for small values of $\mu$ the relative absorption bias has the same order of magnitude as the relative standard deviation, and they are so important that we cannot get reliable estimates without correcting the bias and averaging over many ISIs. In the same range, the error due to a misspecified value of the threshold $S$ is negligible. As $\mu$ increases, both the relative absorption bias and the relative standard deviation decrease to zero (the former as $1 / \mu$ and the latter as $1 / \sqrt{\mu})$ while the error due to misspecification of $S$ settles to $100 \varepsilon / S$.

\section{Ornstein-Uhlenbeck model}

The estimate of $\sigma^{2}$ is practically unbiased. A bias on $\mu$ is detected and in order to quantify it, we plot in Fig. 8 the analogous quantities introduced in the previous sections. In this case the relative bias and standard deviation have been computed from the sample mean and sample variance of estimator (36) averaged over the 10000 simulated trajectories. The relative error introduced if the value $S+\varepsilon$ is erroneously assigned to the threshold level $S$ (with $\varepsilon 10 \%$ of $S$ ) is calculated according to the formula

$$
\begin{aligned}
& \frac{100}{\mu}\left\{\mathcal{M}\left[\hat{\mu}_{a}\left(S+\varepsilon, v_{0}, \beta\right)\right]-\mathcal{M}\left[\hat{\mu}_{a}\left(S, v_{0}, \beta\right)\right]\right\} \\
& \quad=\frac{100 \varepsilon \beta h}{\mu\left(1-\mathrm{e}^{-\beta h}\right)} \mathcal{M}\left(\frac{1}{T}\right),
\end{aligned}
$$
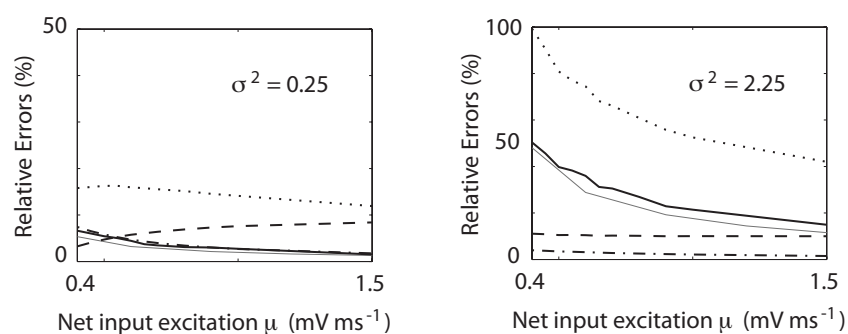

FIG. 8. Relative errors for estimated $\mu$ in the OU process model for two values of $\sigma^{2}=0.25$ and $2.25 \mathrm{mV}^{2} \mathrm{~ms}^{-1}$. Solid line: absorption bias. Dotted line: standard deviation. Dashed line: misspecification of $S$ by $10 \%$. Dash-dotted line: misspecification of $\beta$ by $10 \%$. $S=10 \mathrm{mV}, v_{0}=0 \mathrm{mV}, \beta=0.05 \mathrm{~ms}^{-1}$. where by $\mathcal{M}$ we denote the sample mean. The error due to a wrongly identified time constant is illustrated too. The relative error that is introduced if the value $\beta+\phi$ is erroneously assigned to $\beta$ (let us assume $\phi$ is $10 \%$ of $\beta$ ) is computed as

$$
\frac{100}{\mu}\left\{\mathcal{M}\left[\hat{\mu}_{a}\left(S, v_{0}, \beta+\phi\right)\right]-\mathcal{M}\left[\hat{\mu}_{a}\left(S, v_{0}, \beta\right)\right]\right\} .
$$

For $S$ and $\beta$ respectively fixed to $10 \mathrm{mV}$ and $0.05 \mathrm{~ms}^{-1}$, the computational time required to simulate trajectories with small $\mu$ may become excessively long; hence we consider only values larger than $\mu=0.4 \mathrm{mV} \mathrm{ms}^{-1}$.

As already remarked in Sec. IV D (see Fig. 3), the absorption bias is very well approximated by the expression $\sigma^{2} / S$ holding for a Wiener process with the same parameters, cf. Eq. (33). Hence the plot of the relative bias (Fig. 8, black continuous line) fully overlap the gray line computed according to such a formula.

For small $\sigma^{2}$ (Fig. 8, left panel) and small $\mu$, all the errors have the same order of magnitude, while for increasing $\mu$ the relative absorption bias and the error due to a $10 \%$ misspecified $\beta$ decrease and become negligible with respect to the error due to a $10 \%$ misspecified $S$. For large $\sigma^{2}$ (Fig. 8, right panel), the relative bias due to absorption has the same order of magnitude as the relative standard deviation and they are so important that we need to correct the bias and to consider many ISIs in order to get reliable estimates. The relative error due to misspecification of $S$ by $10 \%$ is constant and comparable with the relative absorption bias only for large values of $\mu$. On the contrary, the relative error due to misspecification of $\beta$ by $10 \%$ is always negligible in the considered range of the parameters. If $\phi$ is negative, the error keeps the same magnitude but in the opposite direction.

\section{CONCLUSIONS}

We showed that the estimators of the net input excitation ( $m=\lambda^{+}-\lambda^{-}$for the RRW model and $\mu$ for the Wiener and OU process models) are biased in integrate-and-fire neuronal models. For both the RRW model and the Wiener process model, we gave analytical formulas for the bias [see Eqs. (33) and (25)], while for the OU process model we estimated the bias from simulated trajectories. We obtained the result that, in the considered range of the parameters, the bias on $\mu$ is very well approximated by the formula that holds for the Wiener process model, with the same parameters (see Fig. 8). In every case considered, the bias is not negligible and it is at least of the same order of magnitude as the errors coming from the misspecification of the known parameters.

On the contrary, the estimate of the net input variance $\left(s^{2}=\lambda^{+}+\lambda^{-}\right.$for the RRW model and $\sigma^{2}$ for the Wiener and OU process models) is biased only for the RRW model, while the bias vanishes for the Wiener and OU process models.

Let us remark that, since the estimate of the net input variance is unbiased in both the Wiener and OU models, we can correct the bias of the estimated net input excitation using in both cases the expression $\sigma^{2} / S$, analytically derived for the Wiener model. 
We could say that the main reason behind the finding of these biases is that the absorption at the threshold changes the process, its distribution, and its expected value at any time. Hence estimators that have been calculated for the process $V_{t}$ in the absence of a threshold do not maintain their properties when computed on a sample drawn from the absorbed process $V_{t}^{a}$.

\section{ACKNOWLEDGMENTS}

This work was supported by Center for Neurosciences LC554, Grant No. AV0Z50110509 and Academy of Sciences of the Czech Republic (Information Society, Grant No. 1ET400110401) and by MIUR (PRIN2005). The authors thank Professor Nico Temme for suggesting the uniform asymptotic expansion of the Bessel function, and the anonymous referees for their helpful comments.

\section{APPENDIX: DIFFUSION LIMIT OF THE FIRST-PASSAGE TIME DENSITY OF THE RRW PROCESS}

Let us prove that under the assumptions of the diffusion limit introduced in Sec. III C, the first-passage time density of the RRW process converges to that of the Wiener model. Let us consider Eq. (4), and take the limit for small amplitudes $a$ of the steps, asking for correspondingly increasing values of the parameters $\lambda^{+}$and $\lambda^{-}$in such a way that $a\left(\lambda^{+}\right.$ $\left.-\lambda^{-}\right)$and $a^{2}\left(\lambda^{+}+\lambda^{-}\right)$respectively tend to to the values $\mu$ and $\sigma^{2}$. The following asymptotics hold: $\lambda^{+} \sim(1 / 2)\left(\sigma^{2} / a^{2}\right.$ $+\mu / a)$ and $\lambda^{-} \sim(1 / 2)\left(\sigma^{2} / a^{2}-\mu / a\right)$. By substituting these expressions into Eq. (4) we get

$$
g_{S}(t)=\frac{S}{t a}\left(\frac{\sigma^{2}+a \mu}{\sigma^{2}-a \mu}\right)^{S / 2 a} \mathrm{e}^{-\sigma^{2} t / a^{2}} I_{S / a}\left(\frac{t}{a^{2}} \sqrt{\sigma^{4}-\mu^{2} a^{2}}\right) .
$$

Due to the uniform asymptotic expansion for large orders of the modified Bessel function (formula 9.7.7 of [22]), we have

$$
I_{S / a}\left(\frac{t}{a^{2}} \sqrt{\sigma^{4}-\mu^{2} a^{2}}\right) \sim \frac{a}{\sqrt{2 \pi t} \sigma} \mathrm{e}^{-S^{2} / t \sigma^{2}+\left(1 / a^{2}\right) \sqrt{a^{2}\left(S^{2}-t^{2} \mu^{2}\right)+t \sigma^{2}}},
$$

and taking the limit of Eq. (A1) using the previous expansion we get Eq. (7).
[1] I. Segev, Trends Neurosci. 15, 414 (1992).

[2] A. Rauch, G. La Camera, H. R. Lüscher, W. Senn, and S. Fusi, J. Neurophysiol. 90, 1598 (2003).

[3] G. La Camera, A. Rauch, D. Thurbon, H. R. Lüscher, W. Senn, and S. Fusi, J. Neurophysiol. 96, 3448 (2006).

[4] R. Jolivet, T. J. Lewis, and W. Gerstner, J. Neurophysiol. 92, 959 (2004).

[5] W. M. Kistler, W. Gerstner, and J. L. vanHemmen, Neural Comput. 9, 1015 (1997).

[6] H. C. Tuckwell, Introduction to Theoretical Neurobiology (Cambridge University Press, Cambridge, U.K., 1988), Vols. 1 and 2.

[7] P. Lansky and S. Sato, J. Peripher Nerv. Syst. 4, 27 (1999).

[8] A. N. Burkitt, Biol. Cybern. 95, 1 (2006).

[9] A. N. Burkitt, Biol. Cybern. 95, 97 (2006).

[10] H. C. Tuckwell and W. Richter, J. Theor. Biol. 71, 167 (1978).

[11] J. Inoue, S. Sato, and L. M. Ricciardi, Biol. Cybern. 73, 209 (1995).

[12] S. Shinomoto, Y. Sakai, and S. Funahashi, Neural Comput. 11, 935 (1999).

[13] S. Ditlevsen and P. Lansky, Phys. Rev. E 71, 011907 (2005).

[14] S. Ditlevsen and P. Lansky, Phys. Rev. E 73, 061910 (2006).

[15] S. Ditlevsen and P. Lansky, Phys. Rev. E 76, 041906 (2007).

[16] B. L. S. Prakasa Rao, Statistical Inference for Diffusion Type Processes (Edward Arnold, London, 1999).

[17] P. Lansky, Math. Biosci. 67, 247 (1983).

[18] P. Lansky, P. Sanda, and J. He, J. Comput. Neurosci. 21, 211
(2006).

[19] R. Höpfner, Math. Biosci. 207, 275 (2007).

[20] M. Sørensen, Int. Statist. Rev. 51, 93 (1983).

[21] B. Ferebee, J. Appl. Probab. 20, 94 (1983).

[22] Handbook of Mathematical Functions with Formulas, Graphs, and Mathematical Tables, edited by M. Abramowitz and I. A. Stegun (U.S. GPO, Washington, DC, 1964).

[23] P. Lansky, J. Theor. Biol. 107, 631 (1984).

[24] L. M. Ricciardi and S. Sato, Lectures in Applied Mathematics and Informatics (Manchester University Press, Manchester, 1990).

[25] A. J. F. Siegert, Phys. Rev. 81, 617 (1951).

[26] R. M. Capocelli and L. M. Ricciardi, Kybernetik 8, 214 (1971).

[27] S. Sato, Math. Biosci. 39, 53 (1978).

[28] L. M. Ricciardi and L. Sacerdote, Biol. Cybern. 35, 1 (1979).

[29] P. Lansky, R. Rodriguez, and L. Sacerdote, Neural Comput. 16, 477 (2004).

[30] W. Feller, An Introduction to Probability Theory and Its Applications (John Wiley \& Sons, New York, 1971), Vol. II.

[31] P. E. Kloeden and E. Platen, Numerical Solution of Stochastic Differential Equations (Springer-Verlag, Berlin, 1992).

[32] M. T. Giraudo and L. Sacerdote, Commun. Stat.-Simul. Comput. 28, 1135 (1999).

[33] P. Lánský and L. Sacerdote, Phys. Lett. A 285, 132 (2001).

[34] D. J. Amit and N. Brunel, Cereb. Cortex 7, 237 (1997). 